\title{
Black-blood dynamic contrast-enhanced carotid artery wall MRI with SRDIR preparation
}

\author{
Zhaoyang Fan ${ }^{1 *}$, Jingsi Xie', Yi He ${ }^{3}$, Yutaka Natsuaki ${ }^{4}$, Ning Jin ${ }^{4}$, Daniel S Berman², Debiao Li ${ }^{1,5}$ \\ From 16th Annual SCMR Scientific Sessions \\ San Francisco, CA, USA. 31 January - 3 February 2013
}

\section{Background}

Inflammation plays a major role in atherosclerotic plaque progression and disruption [1]. Dynamic gadolinium contrast-enhanced (DCE) vessel wall imaging has been used to compute a set of contrast kinetic parameters that may characterize the extent of inflammation of carotid plaques [2-4]. However, previous DCE techniques are limited to a 2D bright-blood acquisition and thus the accuracy of wall signal assessment could be compromised. This work aimed to develop a 3D black-blood DCE technique.

\section{Methods}

\section{Sequence}

An SRDIR (saturation recovery and double inversion recovery) preparation is combined with an RF spoiled gradient-echo sequence to achieve two aims: 1) To create T1-weighting for vessel wall; 2) To consistently null the blood with a fixed inversion time combination (TI1 and TI2) (Fig. 1). Bright-blood acquisition is interleaved with black-blood acquisition to enable arterial blood signal measurement as needed in kinetic modeling.

\section{Protocol}

Nine healthy volunteers (7 F, 2 M; age 31-49 years) were scanned at 3T (Siemens Magnetom Verio) using a 4-channel bilateral carotid coil. The 3D DCE imaging using the SRDIR technique was conducted axially at the carotid bifurcations. Imaging parameters included: resolution $=0.6 \times 0.6 \times 2.0 \mathrm{~mm}^{3}, 4$ partitions, ECG triggering to minimize pulsation motion, 30 lines/RR, TI1/ TI $2=200 / 40 \mathrm{~ms}$ based on computer simulations. Oneframe pre-contrast scan was followed by repetitive contrast-enhanced scans ( $40 \mathrm{~s} /$ frame, $>15 \mathrm{~min})$, along with intravenous contrast $(0.2 \mathrm{mmol} / \mathrm{kg}$ gadopentetate dimeglumine) injection and saline flush $(30 \mathrm{ml})$ both at

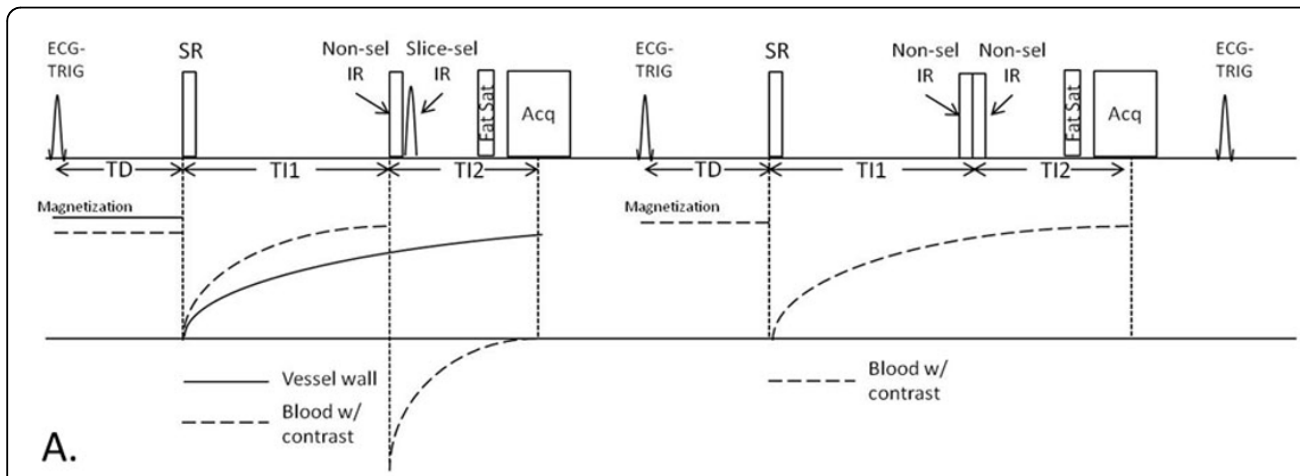

B.

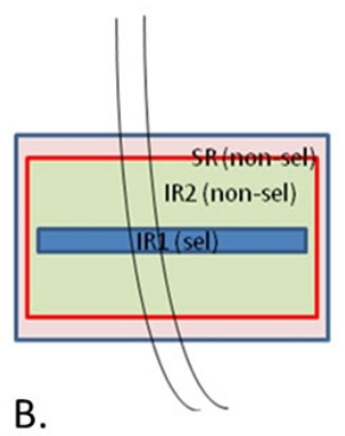

Figure 1 A. Sequence diagram of the SRDIR prepared RF spoiled GRE sequence. B. Locations of SR and DIR pulses.

'Biomedical Imaging Research Institute, Cedars-Sinai Medical Center, Los

Angeles, CA, USA

Full list of author information is available at the end of the article 


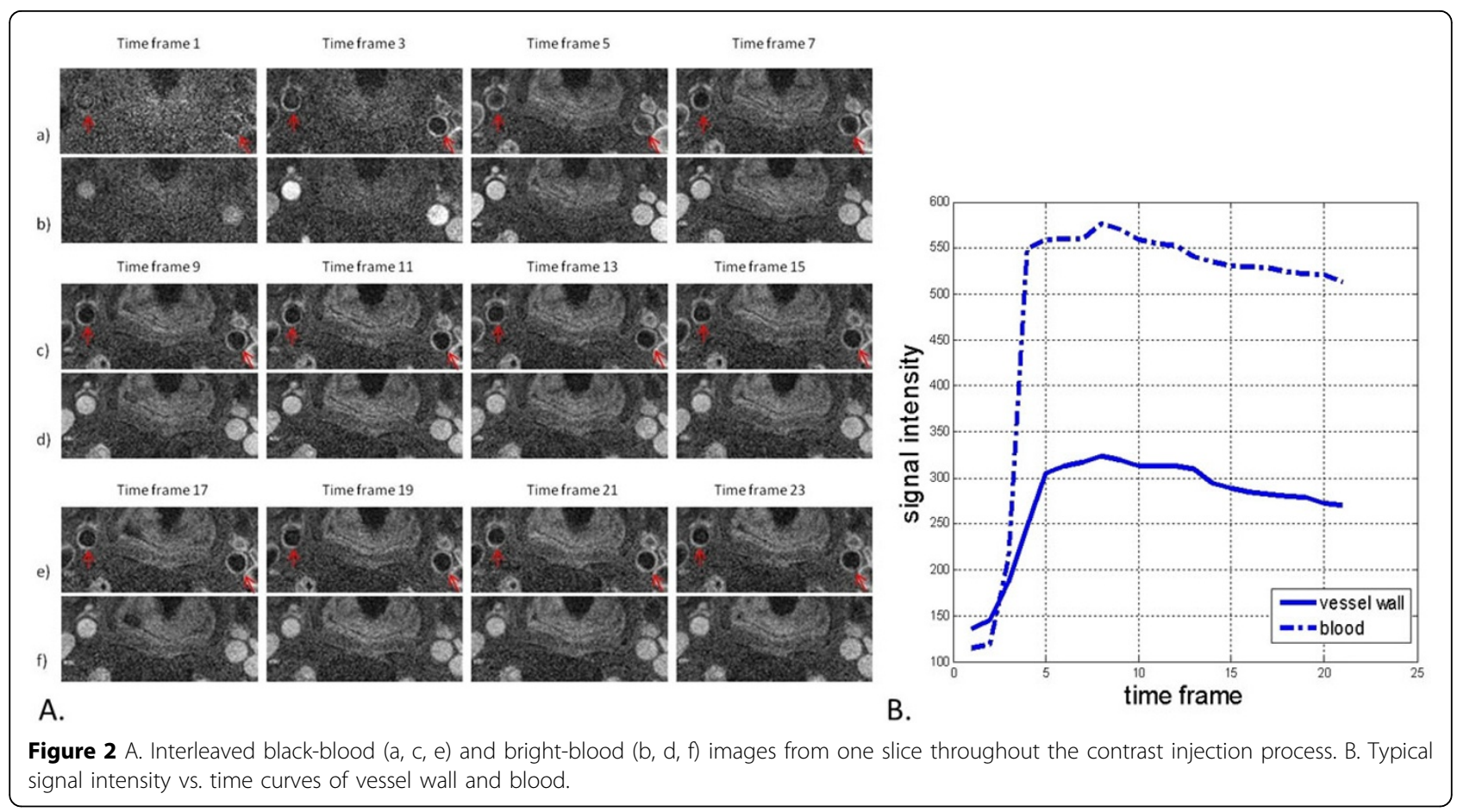

$0.2 \mathrm{ml} / \mathrm{s}$. Through ROI analysis on the black-blood and bright-blood image series, respectively, the changes in signal intensity of carotid artery wall and lumen were obtained and used to compute the kinetic parameters $\left(\mathrm{K}^{\text {trans }}, \mathrm{K}_{\mathrm{ep}}\right.$, and $\mathrm{V}_{\mathrm{p}}$ ) based on Toft's two compartmental model.

\section{Results}

The luminal signal was consistently nulled regardless of the varying blood $\mathrm{T} 1$ values and carotid artery wall were clearly differentiated from the lumen on the DCE images (Fig. 2A). Signal intensity vs. time curves of carotid artery wall and blood were qualitatively similar to previous work (Fig. 2B). From the 9 subjects, $\mathrm{K}^{\text {trans }}=0.062 \pm 0.027 \mathrm{~min}^{-1}$, $\mathrm{K}_{\mathrm{ep}}=0.664 \pm 0.334 \mathrm{~min}^{-1}$, and $\mathrm{V}_{\mathrm{p}}=39.24 \pm 8.34 \%$.

\section{Conclusions}

$\mathrm{K}^{\text {trans }}$ and $\mathrm{V}_{\mathrm{p}}$ values obtained in this work were in accordance with previous studies. To our knowledge, this is the first work in which $K_{e p}$ is investigated for carotid vessel wall. The healthy volunteer data indicates that SRDIR is a promising dynamic carotid vessel wall imaging technique. Clinical validations are currently underway.

\section{Funding}

AHA11POST7650043.

\section{Author details}

'Biomedical Imaging Research Institute, Cedars-Sinai Medical Center, Los Angeles, CA, USA. ${ }^{2}$ Heart Institue and Imaging Center, Cedars-Sinai Medical Center, Los Angeles, CA, USA. ${ }^{3}$ Radiology, Anzhen Hospital, Beijing, China. ${ }^{4}$ Siemens Healthcare, Los Angeles, CA, USA. ${ }^{5}$ Department of Bioengineering, University of California, Los Angeles, CA, USA.

Published: 30 January 2013

doi:10.1186/1532-429X-15-S1-P246

Cite this article as: Fan et al:: Black-blood dynamic contrast-enhanced carotid artery wall MRI with SRDIR preparation. Journal of Cardiovascular Magnetic Resonance 2013 15(Suppl 1):P246.

\section{Submit your next manuscript to BioMed Central and take full advantage of:}

- Convenient online submission

- Thorough peer review

- No space constraints or color figure charges

- Immediate publication on acceptance

- Inclusion in PubMed, CAS, Scopus and Google Scholar

- Research which is freely available for redistribution
Biomed Central 